\title{
Appropriate management of lower respiratory tract infections in primary care
}

\author{
Charles Feldman*
} Division of Pulmonology, Department of Medicine, Johannesburg Hospital and University of the
Witwatersrand, Johannesburg, South Africa

\author{
KEYWORDS \\ General practice; \\ Primary care; \\ Pneumonia; \\ Exacerbations; \\ COPD; \\ Acute bronchitis; \\ LRTIs; \\ Lower respiratory tract \\ infections
}

\begin{abstract}
Summary Respiratory tract symptoms are one of the major reasons for consultations with general practitioners. Most patients present because of symptoms that distress them or their family, or for reassurance that the symptoms do not represent something serious, or in order to receive medication. Many patients believe that their symptoms are due to an infection and expect to receive an antibiotic. These expectations influence the doctor's prescribing such that many patients will receive antibiotics even if the general practitioner considers these not to be required. This article describes the principles of rational antibiotic prescribing for lower respiratory tract infections in primary care, including disorders such as acute bronchitis, acute exacerbations of chronic obstructive pulmonary disease (COPD) and community-acquired pneumonia. (c) 2004 General Practice Airways Group. Published by Elsevier Ltd. All rights reserved.
\end{abstract}

\section{Introduction}

Respiratory tract symptoms area major reason for consultations with a general practitioner and patients presenting with symptoms of an acute lower respiratory tract illness are commonly given antibiotics as part of their treatment [1]. Patients present most commonly because of their symptoms, such as cough, that distress them or those around them, or for reassurance that the symptoms they have are not indicative of something more serious, or in order to receive medication which they consider to be effective [1]; many expect to receive antibiotics [2]. These expectations have a significant influence on the doctors' prescribing,

\footnotetext{
*Present address: Division of Pulmonology, Department of Medicine, University of the Witwatwersrand, Medical School, 7 York Road, Parktown 2193, South Africa. Tel.: +27-11-488-3840; fax: +27-11-488-4675.

E-mail address: feldmanc@medicine.wits.ac.za (C. Feldman).
}

such that many patients will receive antibiotics even when deemed unnecessary [2-4]. In fact, in one investigation conducted among general practitioners to assess their certainty of their decision to treat previously well patients presenting with an acute respiratory tract illness with antibiotics, they considered antibiotics to have been definitely needed in only $19 \%$ of the cases. Furthermore, they acknowledged that antibiotics were probably not indicated or even definitely not indicated in 24 and $1 \%$ of cases, respectively [3].

The term respiratory tract infection represents a wide variety of possible clinical diagnoses, which includes upper and lower respiratory tract infections; the latter including acute bronchitis, acute exacerbations of chronic bronchitis and pneumonia $[1,5]$. Part of the problem in treating patients with respiratory illness is the use of imprecise terms of diagnosis, such as "chest infection" and "bronchitis", both of which imply infections needing antibiotics [2]. However, this has come about because the general practitioner who is presented 


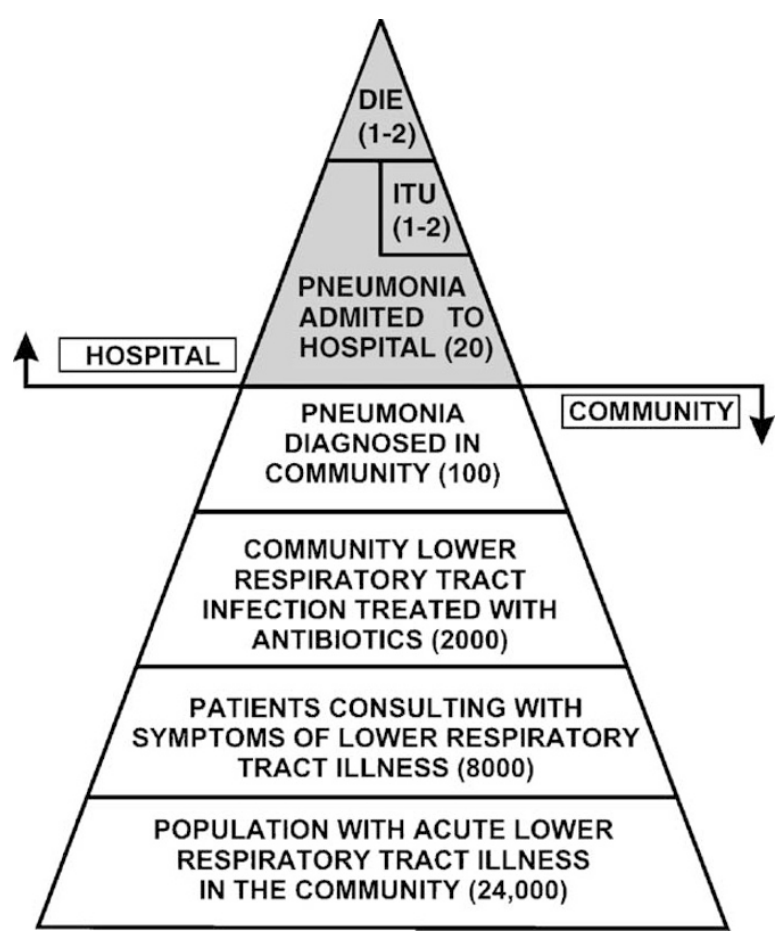

Figure 1 The iceberg of community-acquired respiratory tract illness, infection and pneumonia. Numbers in parenthesis are estimates of relative proportion of patients in each category, based on published definitions and data largely from the UK. Reprinted from Macfarlane J, Lower respiratory tract infection and pneumonia in the community, Semin. Respir. Infect. 14 (1999) 151-162, with permission from Elsevier.

with a patient with respiratory symptoms, is faced with a myriad of possible disorders that represent the pyramid of acute lower respiratory tract illnesses seen in patients in the community (Fig. 1) $[1,6]$. While community-acquired pneumonia, which receives much attention, is a clear-cut disease with specific management guidelines, this infection represents only a small proportion of the respiratory disorders seen by general practitioners [1]. In a recent study of lower respiratory tract infections seen by general practitioners in France, pneumonia represented $9.6 \%$ of diagnoses, exacerbations of COPD $14.9 \%$ and acute bronchitis $72.5 \%$; yet antibiotics were prescribed to $96.5 \%$ of cases [7]. Pneumonia is said to be a cause of some $5-12 \%$ of cases of lower respiratory tract infections in the UK, so that the average British general practitioner would see between 4 and 12 cases per year [8]. However as one goes down the pyramid indicated in the figure it becomes evident that the severity of illness is less, while patient numbers increase considerably, and definitions and classifications of specific illness also become less clear-cut $[1,6]$. Some patients presenting with respira- tory symptoms may even be due to psychological disorders.

Lower respiratory tract infections themselves range in severity from a mild, self-limiting, often viral infection, to a much more severe communityacquired bacterial pneumonia [5]. Thus despite the fact that many of these infections are rather trivial, $50 \%$ of antibiotics used in the community, are for the management of respiratory infections. Some $70 \%$ of patients presenting initially with an acute lower respiratory tract infection are given antibiotics [2,3] and of the $20 \%$ of patients who present again with further or ongoing symptoms, approximately a further $50 \%$ are prescribed antibiotics [1]. The latter is common since symptoms of lower respiratory tract infections are slow to resolve. Patients have often had symptoms for a few weeks before consulting the general practitioner, and typically cough persists for more than 2 weeks in $90 \%$ of cases and more than 3 weeks in $80 \%$ [1].

Thus in most of these cases antibiotic use is neither warranted nor associated with any significant benefit, but certainly does accrue significant health-care costs. Furthermore there is abundant evidence that unnecessary antibiotic use is both potentially harmful to the individual as well as the community, being one of the most important factors associated with the development of resistance among the common respiratory pathogens [2,4]. Much has been discussed and written about what can be done to promote and encourage more conservative and appropriate antibiotic prescribing habits [1], including education of general practitioners and patients. Education should begin with undergraduates and trainees since this early training is said to have a significant influence on their future prescribing practices [1]. Patient education should include information on the types of conditions that would benefit from antibiotic treatment, the natural history of lower respiratory tract infections and symptoms, the evidence for lack of benefit of antibiotics in many conditions, the increasing problem of antibiotic resistance, and the side effects associated with indiscriminate antibiotic use [1]. Patients clearly need to take responsibility for, and be brought into the decision making process about, antibiotic use together with their general practitioner [1].

While GPs need evidence based information on the role and limitations of antibiotics in lower respiratory tract illnesses [1], this needs to be reinforced by information about the natural history of the respiratory illness in the patient [1]. Fig. 2 is a graphic representation of the natural history and durations of symptoms of a patient presenting 


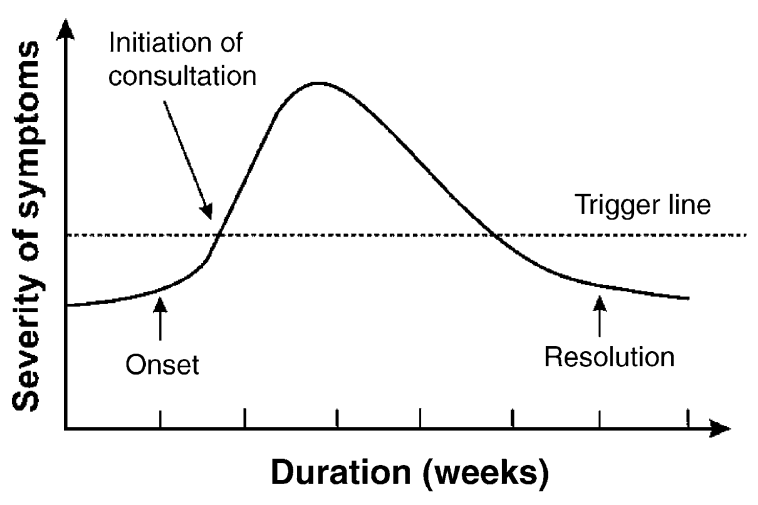

Figure 2 Graphical representation of the natural history and duration of symptoms of lower respiratory tract illness. The "trigger line" represents the level of symptoms below which the individual patient tolerates the symptoms and above which the patient feels "something must be done', Reproduced from Thorax 55 (2000) 153-158, with permission from the Br. Med. J. Publishing Group.

with a lower respiratory tract illness [1]. As shown in the figure, there is a trigger line which crosses the symptom curve and which varies in position from patient to patient. The point at which the patient consults the practitioner and/or purchases medication, is represented where the trigger line crosses this curve. The lower the trigger line the earlier the patient consults [1]. It has been suggested that a better way forward would be to raise the trigger line, i.e. by modifying patients' behaviour and expectations. Informing previously well patients about the natural history of lower respiratory tract infection symptoms does reduce re-consultations and is also tikely to reduce antibiotic prescriptions, as well as future patient consultations [9].

In general practice, this would mean not simply providing a convenient diagnostic label for illness and providing a prescription, but attempting to make as accurate a diagnosis as possible on clinical and if necessary laboratory parameters, and then truly assessing the need for antibiotics and the likelihood that they would be of benefit in any particular case, balancing potential benefits with possible side effects [4]. An important additional aspect of therapy is the appropriate management of symptoms using analgesics, antipyretics, decongestants and bronchodilators, where required [4].

\section{Acute bronchitis}

Acute bronchitis has been described as a recent onset of cough, with sputum production, in a patient with no evidence of underlying chronic obstructive pulmonary disease (COPD), sinusitis or pneumonia [10]. It is a common condition largely diagnosed and managed in general practice, particularly in the winter season [11]. Approximately two million consultations for this condition take place in UK and Wales each year. Most cases, particularly those occurring in previously healthy individuals, are presumed to be viral in origin and very low rates of bacterial isolation have been noted in studies; much lower than that observed in patients with acute exacerbations of COPD [11]. There are also no useful criteria by which to differentiate viral from bacterial causes. Furthermore, it is believed that the majority of these infections do not have any significant effects on lung function and do not contribute to chronic airway obstruction, although in some patients there is a change in forced expiratory volume in one second $\left(\mathrm{FEV}_{1}\right)$ and peak expiratory flow rate (PEF), which improve over 6 weeks, and many of these patient's have, at least transiently, bronchodilator reversibility [11]. For this reason the routine prescription of antibiotics is not recommended as part of initial treatment in primary practice [5]. Nevertheless this does remain a controversial issue and most patients $(60-80 \%)$ both expect, and are given, an antibiotic. This has led to the evaluation of various interventions, including education and bringing the patient into the decision process, which would allow a safe reduction in antibiotic use for acute bronchitis in previously well adults [12].

Cochrane meta-analyses have evaluated the role of antibiotic therapy in the management of patients with acute bronchitis $[5,10,13,14]$. There was evidence for a modest benefit of antibiotic therapy, confirming the impression of many clinicians [14]. However, some of benefits, such as a reduction of mean duration of cough of less than 1 day, were slight, and of doubtful clinical significance. These benefits also need to be counterbalanced by detrimental effects related to costs, potential adverse effects of antibiotics and negative consequences of unnecessary antibiotic use on antibiotic resistance patterns [14]. It is commonly recommended that the decision of whether to use antibiotics in patients with acute bronchitis needs to be shared between patient and clinician, as has been studied in the various interventions, which does decrease overall antibiotic use $[10,14]$. Similar Cochrane analyses have concluded that there is no evidence in upper respiratory tract infections, including common cold and acute purulent rhinitis in children and adults, of any benefit of antibiotic therapy, and there is a significant increase of adverse effects in adults [15]. 


\section{Acute exacerbations of chronic obstructive pulmonary disease (COPD)}

"COPD is a disease state characterized by airflow limitation that is not fully reversible. The airflow limitation is usually both progressive and associated with an abnormal inflammatory response of the lungs to noxious particles or gases" [16]. COPD occurs predominantly as a consequence of cigarette smoking and is associated with an exaggerated decline in lung function. The condition is punctuated by acute exacerbations that are precipitated in a substantial number of cases by infection of the airway [16-18]. Infections, together with environmental exposure are said to be the common precipitating factors of acute exacerbations [16,18-20]. Depending on study designs, up to $50 \%$ of acute exacerbations are found to be due to bacterial infections. In addition to being associated with increased morbidity and mortality and health-care costs, it is now also recognized that these acute exacerbations may also accelerate the decline in lung function [17].

Importantly, there is also emerging evidence of a significant relationship between lower airway bacterial colonization and disease progression in COPD. The lower airway is normally sterile, but many patients with COPD become colonized in the lower airway with bacteria [20]. In one study, the rate of decline in lung function, as measured by $\mathrm{FEV}_{1}$, was proportional to the rise in bacterial colonising load, the latter appearing to be even more important in the rapid decline in lung function than current cigarette smoking in the patients [21].

One problem in evaluating studies of acute exăc erbations is that there is no universal definition of the condition [5]. Most commonly, exacerbations are defined according to the presence of symptoms, the most important ones being increasing shortness of breath, increasing sputum volume and increasing sputum purulence $[5,16]$. Those patients with all three symptoms have a severe exacerbation [5]. Sputum purulence (green sputum) is a very important indicator of the possible presence of bacterial infection as a precipitating factor for the acute exacerbation [22]. Studies have shown that increasing sputum purulence correlates well with the presence of airway bacterial infection, as well as with the presence of cytokine markers of airway inflammation [23]. Therefore patients with purulent sputum are in more need of, and more likely to benefit from, antibiotic therapy during exacerbations.

A number of reviews have been written and various studies have been undertaken, investigating the optimal therapy of acute exacerbations of COPD $[17,18,24]$. Meta-analyses of these stud- ies have appeared in the Cochrane database of evidenced based medicine and do give some indication of best therapy. In particular, more research is clearly needed in the role of antibiotics in acute exacerbations.

\section{(1) Bronchodilators $[16,19,25,26]$}

Short acting bronchodilators, i.e. $\beta_{2}$-agonists and anticholinergics, are the mainstay of therapy for acute exacerbations. There is evidence that both have equal effects on spirometric parameters, and although they are most commonly used in combination, there are marginal benefits of this combination. Nevertheless this is still recommended by most physicians. There has been some suggestion that side effects overall may be somewhat less with the use of anticholinergics. Initiation of therapy with methylxanthines is not routinely recommended, because of potential toxicity, and because studies investigating whether they have added benefits are equivocal. They may be considered in patients who do not respond to other bronchodilators. However, if the patient is already on these agents they should be continued. Drug interactions with methylxanthines and other agents used in COPD (e.g. some of the antibiotics) need to be considered. There does not appear to be any evidence of benefit of one delivery device over another (whether metered dose inhater or nebuliser) and so patient preference and ability is considered in this choice.

(2) Corticosteroids [16, 19,27]

A short course of corticosteroids of up to 2 weeks is associated with improved spirometry and may be associated with a shorter hospital stay. There is evidence that their use may also decrease the relapse rate in COPD. The optimal route, dose and duration are uncertain.

(3) Antibiotic therapy $[5,16,17,19,20,28]$

One of the more controversial issues in the management of acute exacerbations of COPD has been the value of, and indications for, antibiotic therapy. In general terms based on more recent studies, antibiotics are beneficial in patients with acute exacerbations of COPD. Patients with more severe underlying COPD and those with more severe exacerbations are most likely to benefit. Indications for antibiotics in the management of acute exacerbations of COPD are shown in Table 1. Patients with less severe COPD are most commonly infected with the usual respiratory pathogens including Streptococcus pneumoniae, Haemophilus influenzae, and Moraxella catarrhalis [5]. 
Table 1 Patients with acute exacerbations of COPD who should be treated with antibiotics (reproduced with permission from S. Afr. Respir. J., 2003 [17]).

More severe underlying COPD

Severe exacerbations

Purulent (green) sputum during exacerbations

Evidence of systemic infection (e.g. fever, raised

white cell count, raised activity markers)

New infiltrate on chest radiograph

Presence of pneumonia

$H$. influenzae is particularly common in patients who still continue to smoke. Viral infections account for approximately $20-30 \%$ of cases. Infections with the so-called "atypical pathogens", such as Chlamydia pneumoniae, Mycoplasma pneumoniae and Legionella spp., account for a small percentage of infections [5]. Antibiotics directed against these common bacterial pathogens should be effective in cases with less severe COPD and the exact antimicrobial agents chosen would depend on local patterns of antibiotic resistance among these organisms. It is only in severe cases of COPD and particularly in patients with an FEV 1 less than $50 \%$ predicted (and especially $<35 \%$ ) that gram-negative Enterobacteriaciae (for example, Klebsiella pneumoniae) and Pseudomonas aeruginosa might be found, requiring additional and specific antibiotic therapy [5].

Very few of the currently published guidelines for the management of COPD have any clear indications of which particular class of antibiotics should be prescribed in any particular situation. The first published guidelines that have a more comprehensive description of antibiotic therapy are the Canadian and South African guidelines (in press) $[17,28]$. These guidelines divide patients with COPD into those with "simple" or "complicated" COPD or exacerbations $[17,28]$. The former have no risk factors for treatment failure or infection with resistant pathogens and therapy with some of the more standard, inexpensive antibiotics is recommended. Among the recommendations include amoxicillin, amoxicillin/clavulanate, doxycycline, newer macrolides/azalide, and ketolides $[17,28]$. The group of patients with complicated exacerbations is more likely to fail antibiotic therapy and has risk factors for infection with more virulent or resistant pathogens. Here antibiotic choices may include amoxicillin/clavulanate, second or third generation cephalosporins, or a new "respiratory" fluo- roquinolone $[17,28]$. The optimal duration of antibiotic therapy is uncertain. Most clinicians prescribe 5-10 days of antibiotic treatment and this seems to be appropriate. Antibiotic therapy of acute exacerbations has been shown to decrease the relapse rate of COPD $[20,29]$.

(4) Oxygen $[16,19]$

Oxygen therapy provides significant benefit to hypoxaemic patients with COPD. The major concern is the possibility of the development of hypercarbia, with the occurrence of respiratory failure, and this needs to be monitored using blood gas analysis.

(5) Mucus clearance strategies [30]

The commonly held belief is that mucus clearance strategies do not shorten the course of exacerbations although there may be some improvement of symptoms and a small reduction in acute exacerbations. Mechanical percussion of the chest is ineffective and may even be detrimental in acute exacerbations.

(6) Additional aspects of treatment [16]

Additional therapy should include monitoring of fluid balance and nutrition, identification and treatment of associated conditions (e.g. cardiac failure), and prophylaxis for deep venous thrombosis. Indications for hospitalisation, need for ICU admission and/or ventilatory support should be considered and are beyond the scope of this article.

(7) Preventative strategies [24]

"One of the most important interventions that can be undertaken is to encourage smoking cessation in patients who continue to smoke. This is one of the only interventions that may alter the natural course of the disease. Pneumococcal and influenza vaccination are strongly recommended and may be associated with a significant decrease in infectious complications. Lastly patient education and rehabilitation are essential components of effective management.

\section{Community-acquired pneumonia}

Community-acquired pneumonia is an important cause of considerable morbidity and mortality, and need for hospitalisation in patients worldwide $[8,31]$. It occurs with a frequency of approximately $1-3$ per 1000 adults per year in the developed world, with an estimated worldwide incidence of 10 million cases annually, with a mortality rate as high as $50 \%$ in seriously ill patients and in high-risk cases [32]. A study from the UK suggested that each general practitioner sees on average about 10 
cases a year and $80 \%$ of these cases are managed at home [33]. The frequency of pneumonia is higher at extremes of age and in high-risk patients with underlying predisposing conditions, particularly disorders such as COPD [31,34]. The latter is particularly important because of its association with cigarette smoking, which is the main avoidable risk factor for community-acquired pneumonia in adults [34].

The mainstay of therapy is antibiotic treatment, which is required in all cases [35]. There is emerging evidence that the prognosis of pneumonia is improved by the early initiation of antibiotics to which the causative organisms are susceptible and adversely affected by in appropriate or delayed treatment. Numerous guidelines have been published internationally describing the optimal treatment of pneumonia in the various regions. These guidelines are usually based on published evidence as well as opinion, but also take into account local factors. Therefore despite similarities among the various guidelines, there are also significant differences and it is for this reason that a universal guideline that would satisfy all areas cannot be developed. Initial antimicrobial therapy of pneumonia is of necessity empiric, since it is a serious infection requiring urgent treatment and the causative pathogens are not usually known at the time of initial diagnosis [32]. Antibiotic treatment decisions are hampered throughout the world by emerging antimicrobial resistance among the common respiratory pathogens $[31,32,35]$.

Nevertheless there are certain universal principles of antimicrobial therapy of pneumonia that should be applied in all cases and which should en sure optimal therapy with best possible outcome. In the first instance the diagnosis of pneumonia needs to be confirmed, which is not always straightforward [8]. As discussed above, many patients are treated unnecessary with antibiotics simply on the suspicion of the presence of pneumonia, or other lower respiratory tract infection, without confirmation of the diagnosis. Symptoms at presentation also do not distinguish pneumonia from other causes of respiratory illnesses. While the presence of focal features signs such as crackles and/or evidence of consolidation on clinical examination is very suggestive of the presence of pneumonia, only chest radiography, with the demonstration of a lobar or segmental shadowing not due to another cause is a reasonably reliable indicator of the presence of pneumonia, and has been considered a gold standard of diagnosis $[5,8,32,33,35,36]$. It is for this reason that most published guidelines recommend chest radiology for cases suspected of having pneumonia, particularly in the case of those patients that are sick enough to be admitted to hospital [37].

Clinical assessment of the patient should include elucidation of any risk factors for pneumonia, of any clues to possible aetiology and an evaluation of the severity of the infection [5,31,32,35-38]. This includes information on the age of the individual and evidence of underlying co-morbid illnesses, which are known to be associated with an increased mortality. Evaluation of the severity of the pneumonia is important for a number of reasons, such as decisions as to whether the patient should be admitted to hospital or could be safely managed at home, guidance as to what investigations need to be performed, assessment of the need for admission to the intensive care unit and the likely prognosis, and also as a guide to the choice of initial antibiotic treatment $[32,36]$. A number of severity scoring systems or indicators have been developed for patients with pneumonia, such as the Pneumonia Severity Index (PSI), the British Thoracic Society (BTS) "rule" and the American Thoracic Society criteria [36-39]. Recently described and tested has been the CURB-65 severity prediction tool, which is based on the modified BTS "rule" [39]. Using this rule the CURB severity score was calculated (parameters used are confusion, blood urea $>7 \mathrm{mmol} / \mathrm{l}$, respiratory rate $\geq 30$ breaths $/ \mathrm{min}$ and low blood pressure). Added to that is age $\geq 65$ years, which is an independent predictor of outcome, and allows a CURB-65 score to be derived. Although it appears promising it needs further validation and if found to be relatively accurate, its simplicity and ease could prove to be very valuable [39]. Currently, while severity indicators are useful adjuncts they cannot replace accurate clinical evaluation.

It is also important, as part of the assessment, for the clinician to be aware of patient allergy to any antibiotics, as well have information as to whether the patient has had any previous antibiotic treatment in the recent past, both of which may exclude the use certain agents. Perhaps most importantly, clinicians managing patients with pneumonia need to have a thorough knowledge of the pathogens commonly seen in the local region and their antimicrobial susceptibility patterns [5].

Outpatient treatment of pneumonia is usually undertaken with one of the oral antibiotics to which the common pathogens are known to be susceptible, for example, high-dose amoxicillin or the macrolides/azalide $[32,37]$. For inpatient's, the antibiotic therapy most often recommended is that of a combination of a beta-lactam and macrolide/azalide [32,37]. There is considerable emerging evidence that in sicker, hospitalized cases with pneumonia, including patients with 
bacteraemic pneumococcal pneumonia, this combination treatment is associated with a better outcome than in patients receiving monotherapy $[37,40,41]$. The reasons that combining a macrolide/azalide with a beta-lactam has added benefit is uncertain, but could include one or more of the following factors; additional antibiotic cover for so-called "atypical pathogens", which require treatment with a macrolide/azalide; additional antibiotic cover for beta-lactam resistant pathogens that are sensitive to macrolides; synergistic antimicrobial activity of benefit in sicker, high risk or immunocompromised patients; and anti-inflammatory, immunomodulatory activity of macrolides/azalide [40].

Importantly, antibiotic therapy needs to be commenced as soon as possible after diagnosis of pneumonia, with evidence that initiation of treatment within $8 \mathrm{~h}$ or even less is associated with better 30-day mortality [42]. There is also no evidence that prolonging antibiotic treatment has any advantage but rather may be associated with emergence of resistance. It is usually recommended that treatment be continued for 5-10 days in routine cases, with longer treatment advised for infections with the "atypical pathogens" [32,37]. Other aspects of treatment, beyond antibiotics, also need to be considered and include maintenance of nutrition and hydration, correction of hypoxaemia using supplemental oxygen, adequate analgesia for pleuritic chest pain and appropriate management of $[31,32,37]$ underlying co-morbid conditions, such as COPD.

Prevention of pneumonia is extremety important and includes control of undertying co-morbid factors, including smoking and alcohol abuse, which are known to be important risk factors $[32,35]$. Use of influenza and pneumococcal vaccines for appropriate cases as recommended in the local guidelines is also associated with a decrease in the occurrence of pneumonia and its complications $[31,32,35,37]$.

\section{References}

[1] Macfarlane J, Holmes WF, Macfarlane R. Do hospital physicians have a role in reducing antibiotic prescribing in the community? Thorax 2000;55:153-8.

[2] Macfarlane J, Holmes W, Macfarlane R, Britten N. Influence of patients' expectations on antibiotic management of acute lower respiratory tract illness in general practice: questionnaire study. Br Med J 1997;315:1211-4.

[3] Macfarlane J, Lewis SA, Macfarlane R, Holmes W. Contemporary use of antibiotics in 1089 adults presenting with acute lower respiratory tract illness in general practice in the UK: implications for developing management guidelines. Respir Med 1997;91:427-34.
[4] Colgan R, Powers JH. Appropriate antimicrobial prescribing: approaches that limit antibiotic resistance. Am Fam Physician 2001;64:999-1004.

[5] Ortqvist A. Treatment of community-acquired lower respiratory tract infections in adults. Eur Respir J 2002;20(Suppl 36): $40 \mathrm{~s}-53 \mathrm{~s}$.

[6] Macfarlane J. Lower respiratory tract infection and pneumonia in the community. Semin Respir Infect 1999;14:15162.

[7] Raherison C, Peray P, Poirier R, Romand P, Grignet J-P, Arsac $P$, et al. Management of lower respiratory tract infections by French general practitioners: the AIR II study. Eur Respir J 2002;19:314-9.

[8] Holmes WF, Woodhead M. Providing better care for patients who may have pneumonia. Thorax 1999;54:925-8.

[9] Macfarlane JT, Holmes WF, Macfarlane RM. Reducing reconsultations for acute lower respiratory tract illness with an information leaflet: a randomized controlled study of patients in primary care. Br J Gen Pract 1997;47:71922.

[10] Chandran R. Should we prescribe antibiotics for acute bronchitis? Am Fam Physician 2001;64:135-8.

[11] Boldy DAR, Skidmore SJ, Ayres JG. Acute bronchitis in the community: clinical features, infective factors, changes in pulmonary function and bronchial reactivity to histamine. Respir Med 1990;84:377-85.

[12] Macfarlane J, Holmes W, Gard P, Thornhill D, Macfarlane R, Hubbard R. Reducing antibiotic use for acute bronchitis in primary care: blinded, randomized controlled trial of patient information leaflet. Br Med J 2002;12:91-4.

[13] Becker L, Glazier R, Mclsaac W, Smucny J. Antibiotics for acute bronchitis. In: The Cochrane library, Issue 3. Oxford: Update Software; 1999 [Cochrane review].

[14] Smucny J, Fahey T, Becker L, Glazier R. Antibiotics for acute bronchitis. In: The Cochrane library, Issue 4. Chichester, UK: Wiley; 2003 [Cochrane review].

[15] Arroll B, Kenealy T. Antibiotics for the common cold and acute purulent rhinitis. In: The Cochrane library, Issue 4. Chichester, UK: Wiley; 2003 [Cochrane review].

416] National Institutes of Health NHLBI. Global Initiative for Chronic Obstructive Lung Disease. Global Strategy for the Diagnosis, Management, and Prevention of Chronic Obstructive Pulmonary Disease. http://www.goldcopd.com/; 2003. Last accessed on 9 January 2004.

[17] Feldman C. Acute exacerbations of chronic obstructive pulmonary disease. Guidelines for antibiotic management. SA Respir J 2003;9:44-51.

[18] Stoller JK. Acute exacerbations of chronic obstructive pulmonary disease. N Engl J Med 2002;236:988-94.

[19] Hunter MH, King DE. COPD: management of acute exacerbations and chronic stable disease. Am Fam Physician 2001;64:603-12.

[20] Adams SG, Melo J, Luther M, Anzueto A. Antibiotics are associated with lower relapse rates in outpatients with acute exacerbations of COPD. Chest 2000;117:1345-52.

[21] Wilkinson TMA, Patel IS, Wilks M, Donaldson GC, Wedwicha JA. Airway bacterial load and $\mathrm{FEV}_{1}$ decline in patients with chronic obstructive pulmonary disease. Am J Respir Crit Care Med 2003;167:1090-5.

[22] Stockley RA, O'Brien C, Pye A, Hill SL. Relationship of sputum color to nature and outpatient management of acute exacerbations of COPD. Chest 2000;117:1638-45.

[23] Gompertz S, O'Brien C, Bayley DL, Hill SL, Stockley RA. Changes in bronchial inflammation during acute exacerbations of chronic bronchitis. Eur Respir J 2001;17:1112-9.

[24] Heath JM, Mongia R. Chronic bronchitis: primary care management. Am Fam Physician 1998;57:2365-72, 2376-8. 
[25] Brown CD, McCrory D, White J. Inhaled short-acting beta $_{2}$-agonists versus ipratropium for acute exacerbations of chronic obstructive pulmonary disease. In: The Cochrane library, Issue 4. Chichester, UK: Wiley; 2003 [Cochrane review].

[26] Barr RG, Rowe BH, Camargo Jr CA. Methylxanthines for exacerbations of chronic obstructive pulmonary disease. In: The Cochrane library, Issue 2. Oxford: Update Software; 2002 [Cochrane review].

[27] Wood-Baker R, Walters EH, Gibson P. Oral corticosteroids for acute exacerbations of chronic obstructive pulmonary disease. In: The Cochrane library, Issue 2. Oxford: Update Software; 2002 [Cochrane review].

[28] O'Donnell DE, Aaron S, Bourbeau J, et al. Canadian Thoracic Society recommendations for management of chronic obstructive pulmonary disease-2003. Can Respir J 2003;10(Suppl A):11A-33A.

[29] Kirchner JT. Do antibiotics reduce relapse of COPD exacerbations? Am Fam Physician 2000;62:2101.

[30] Poole PJ, Black PN. Mucolytic agents for chronic bronchitis. In: The Cochrane library, Issue 2. Oxford: Update Software; 2002 [Cochrane review].

[31] Tsirgiotis E, Ruffin R. Community-acquired pneumonia. A perspective for general practice. Aust Fam Physician 2000;29:639-45.

[32] Finch R. Community acquired pneumonia. J R Coll Physicians 1998; 32:328-32.

[33] Woodhead MA, Macfarlane JT, McCracken JS, Rose DH, Finch RG. Prospective study of the aetiology and outcome of pneumonia in the community. Lancet 1987;21:6714.

[34] Farr BM, Woodhead MA, Macfarlane JT, Bartlett CL, MCCracken JS, Wadsworth J. Risk factors for communityacquired pneumonia diagnosed by general practitioners in the community. Respir Med 2000;94:422-7.

[35] File Jr TM. Community-acquired pneumonia. Lancet 2003; 362:1991-2001.

[36] Metlay JP, Fine MJ. Testing strategies in the initial management of patients with community-acquired pneumonia. Ann Intern Med 2003;138:109-18.

[37] BTS guidelines for the management of community-acquired pneumonia in adults. Thorax 2001;56(Suppl IV):1-64.

[38] Halm EA, Teirstein AS. Management of community-acquired pneumonia. N Engl J Med 2002;347:2039-45.

[39] Woodhead M. Assessment of illness severity in communityacquired pneumonia: a useful new prediction tool? Thorax 2003;58:371-2.

[40] Waterer GW, Somes GW, Wunderink RG. Monotherapy my be suboptimal for severe bacteremic pneumococcal pneumonia. Arch Intern Med 2001;161:1837-42.

[41] Gleason PP, Meehan TP, Fine JM, Galusha DH, Fine MJ. Associations between initial antimicrobial therapy and medical outcomes for hospitalized elderly patients with pneumonia. Arch Intern Med 1999;159:2511-2.

[42] Meehan TP, Fine MJ, Krumholz HM, Scinto JD, Galusha $\mathrm{DH}$, Mockalis JT, et al. Quality of care, process, and outcomes in elderly patients with pneumonia. J Am Med Assoc 1997;278:2080-4.
Available online at www.sciencedirect.com

science@Direct 